\title{
Kernel Inference on the Generalized Gamma Distribution Based on Generalized Order Statistics
}

\author{
M. Ahsanullah ${ }^{1}$ \\ M. Maswadah ${ }^{2}$ and Seham $^{3}$ Ali M. \\ Received 6 January 2013 \\ Accepted 16 March 2013
}

\begin{abstract}
The kernel approach has been applied using the adaptive kernel density estimation, to inference on the generalized gamma distribution parameters, based on the generalized order statistics (GOS). For measuring the performance of this approach comparing to the Asymptotic Maximum likelihood estimation, the confidence intervals of the unknown parameters have been studied, via Monte Carlo simulations, based on their covering rates, standard errors and the average lengths. The simulation results indicated that the confidence intervals based on the kernel approach compete and outperform the classical ones. Finally, a numerical example is given to illustrate the proposed approaches developed in this paper.
\end{abstract}

${ }^{1}$ Department of Management Sciences, Rider University,

Lawrenceville, NJ 08648-3099, USA

E-mail: ahsan@rider.edu

2,3 Department of Mathematics, Faculty of science, Aswan university, Aswan, Egypt

E-mail: maswadah@hotmail.com E-mail:seham_1elwany@yahoo.com 
Keywords and Phrases: Generalized gamma distribution; Generalized order statistics; Maximum likelihood estimation; Kernel density estimation; Asymptotic maximum likelihood estimations.

\section{Introduction}

A random variable $X$ is said to have generalized gamma distribution (GGD), if its probability density function (PDF) has the form:

$$
f(x ; \alpha, \beta, \lambda)=\frac{\alpha}{\Gamma(\lambda)} \beta^{-\alpha \lambda_{x} \alpha \lambda-1} \exp \left[-(x / \beta)^{\alpha}\right], \quad x>0 \alpha, \beta, \lambda>0,
$$

where $\Gamma(\lambda)$ is gamma function, $\alpha, \beta$ and $\lambda$ are the shape, scale and index parameters respectively. The corresponding cumulative distribution function (CDF) is given by:

$$
F(x ; \alpha, \beta, \lambda)=\frac{I G\left(\lambda,(x / \beta)^{\alpha}\right)}{\Gamma(\lambda)}, \quad x>0
$$

where $I G\left(\lambda,(x / \beta)^{\alpha}\right)=\int_{0}^{(x / \beta)^{\alpha}} t^{\lambda-1} e^{-t} d t, \quad$ is the lower incomplete gamma function.

Stacy (1962) introduced the GGD, which offers a highly flexible family of life testing models that includes a considerable number of distributions as special cases, namely, the exponential distribution $(\lambda=\alpha=1)$, gamma distribution $(\alpha=1)$ and Weibull distribution $(\lambda=1)$. The lognormal distribution is also obtained as a limiting distribution when $\lambda \rightarrow \infty$.

The statistical analysis of the GGD based on complete as well as censored samples have been studied by many authors such as Stacy and Mihram (1965), Parr and Webter (1965), Harter (1967), Hager and Bain (1970), Prentice (1974), Lawless (1980), Di Ciccio (1987), Wingo (1987), Wong (1993), Cohen and Whitten (1988) 
and Maswadah (1989, 1991). Hwang and Huang (2006) introduced a new moment estimation for the generalized gamma distribution parameters using its characterization. Dadpay et al. (2007) introduced some concepts of the GGD, via information theory. Gomes et al. (2008) used the ML method for estimating the parameters by justifying the model in terms of a simpler alternative form. Geng and Yuhlong (2009) proposed a new parameterization of the GGD to sustain the numerical stability for the maximum likelihood estimation based on the progressively type-II censored sample. Mukherjee et al. (2011) presented a Bayesian study for the generalized gamma model.

In this paper, the kernel density estimation has been applied for deriving the confidence intervals for the unknown parameters of the GGD comparing to the asymptotic maximum likelihood estimator based on the GOS, that introduced by Kamps (1995) as a unified model that includes several models of ordered random variables, such as ordinary order statistics, type-II censored order statistics, progressively type-II censored order statistics, record values and sequential order statistics. For more details about the generalized order statistics, see Ahsanullah (1995, 2000).

Let $X(1, n, \tilde{m}, k), \ldots, X(n, n, \tilde{m}, k),(k \geq 1, \tilde{m}>-1$ is a real number) be $n$ generalized order statistics from a continuous population with $\operatorname{CDF} F(x)$ and PDF $f(x)$, thus their joint PDF has the form:

$$
\begin{aligned}
f_{X(1, n, \tilde{m}, k), \ldots,} & X(n, n, \tilde{m}, k) \\
& \left(x_{1}, x_{2}, \ldots, x_{n}\right)=k \prod_{i=1}^{n-1} \gamma_{i} f\left(x_{i}\right)\left[1-F\left(x_{i}\right)\right]^{m} \\
& \times\left[1-F\left(x_{n}\right)\right]^{k-1} f\left(x_{n}\right),
\end{aligned}
$$

on the cone $F^{-1}(0)<x_{1}<\ldots<x_{n}<F^{-1}(1)$ of $R^{n}$,

where 


$$
\begin{aligned}
& \tilde{m}=\left(m_{1}, m_{2}, \ldots, m_{n-1}\right) \in R^{n-1}, \gamma_{r}=k+(n-r)+M_{r}>0, \text { such that } \\
& M_{r}=\sum_{j=r}^{n-1} m_{j}, \gamma_{n}=k>0
\end{aligned}
$$

Particular cases from (2):

1- Ordinary order statistics: for $k=1$ and $\tilde{m}=0$.

2- Type II right censored order statistics: for $k=1$ and $m_{i}=0, i=1,2, \ldots, n-1$,

$$
m_{n}=n-r \text {. }
$$

3- Type II progressive censored order statistics: for $m_{i} \neq 0, i=1,2, \ldots, n-1$,

$$
m_{n}=k-1 \text {. }
$$

4- Record values for $k=1$ and $\tilde{m}=-1$.

\section{Main Results}

\subsection{Kernel Estimation}

In this section, we apply the unconditional approach for deriving the confidence intervals to the unknown parameters based on the adaptive kernel density estimation (AKDE), which is asymptotically converged to any density function depending only on a random sample, though the underlying distribution is not known. This approach has been applied for some distributions, see Maswadah (2006, 2007). In the univariate case, the adaptive kernel density estimation based on a random sample of size $n$ from the random variable $X$ with unknown probability density function $f(x)$ and support on $(0, \infty)$ is given by: 


$$
\hat{f}(x)=\frac{1}{n} \sum_{i=1}^{n} \frac{1}{h_{i}} K\left(\frac{x-x_{i}}{h_{i}}\right),
$$

where $h_{i}=h \lambda_{i}$ and $\lambda_{i}$ is a local bandwidth factor which narrows the bandwidth near the modes and widens it in the tails, which can be defined as:

$$
\lambda_{i}=\left(\frac{G}{\hat{f}\left(x_{i}\right)}\right)^{0.5}
$$

where $G$ is the geometric mean of the $\hat{f}\left(x_{i}\right), i=1,2, \ldots, n$ and $h$ is a fixed (pilot) bandwidth. We can see that our estimate $\hat{f}(x)$ is bin-independent regardless of our choice of $K$, where the role of $K$ is to spread out the contribution of each data point in our estimate of the parent distribution, that controls the shape. The most important part in the kernel estimation method is to select the bandwidth (scaling) or the smoothing parameter, thus its selection has been studied by many authors, see Abramson (1982) and Guillamon et al. (1998) based on minimizing the mean square errors, however, the optimal choice in most cases is $h=1.059 \cdot S \cdot n^{-0.2}$, where $S$ is the sample standard deviation and we will consider it as the pilot bandwidth. However, it must be mentioned that the optimal choice $h$ can't possibly be optimal in every application, and its choice is really depended on the application under consideration to different bandwidths. Though, there is a variety of kernel functions with different properties have been used in literature, however, the obvious and natural choice of the kernel functions is the standard Gaussian kernel, for its continuity, differentiability, and locality properties.

The kernel approach depending on finding the kernel density estimation for pivotal random variables, that depending on the unknown parameters and whose distributions are free of unknown parameters. For the GGD (1), $\left(x_{i} / \beta\right)^{\alpha}, \ldots,\left(x_{n} / \beta\right)^{\alpha}$ be a sample of size $n$ from the gamma distribution $G(\lambda, 1)$, thus if $\hat{\alpha}$ and $\hat{\beta}$ are the MLEs of $\alpha$ and $\beta$ respectively, then 


$$
\left(x_{i} / \beta\right)^{\alpha}=\left(\left(x_{i} / \beta\right)^{\hat{\alpha}}\right)^{\alpha / \hat{\alpha}}=\left[(\hat{\beta} / \beta)^{\hat{\alpha}} \cdot\left(x_{i} / \hat{\beta}\right)^{\hat{\alpha}}\right]^{\alpha / \hat{\alpha}}=\left[a_{i} z_{2}\right]^{z_{1}},
$$

for $i=1,2, \ldots, n$, are independent of the unknown parameters $\alpha$ and $\beta$ when $\lambda$ is known. Therefore, $Z_{1}=\alpha / \hat{\alpha}$ and $Z_{2}=(\hat{\beta} / \beta)^{\hat{\alpha}}$ are pivotal quantities and $a_{i}=\left(x_{i} / \hat{\beta}\right)^{\hat{\alpha}}, i=1,2, \ldots, n$ form a set of ancillary statistics. Note that the ancillary statistics satisfy the maximum likelihood equations, therefore, any $n-2$ of $a_{i}{ }^{\prime} s$, say $a_{1}, \ldots, a_{n-2}$ form a set of $n-2$ functionally independent ancillary statistics. For utilizing the kernel function for estimating the probability density function (PDF) of a pivotal, we can summarize the method in the following algorithm:

1- Let $\left(x_{1}, x_{2}, \ldots, x_{n}\right)$ be a random sample of size $n$ from the random variable $X$, with $\operatorname{PDF} f(x ; \theta)$, where $\theta$ represents the unknown parameter with support on $(0, \infty)$.

2- Bootstrapping with replacement n samples $X_{i}^{*}$ of size $n$ from the parent sample in step 1 , where $X_{i}^{*}=\left(x_{1}^{*}, x_{1}^{*}, \ldots, x_{n}^{*}\right), i=1,2, \ldots, n$.

3- For each sample in step 2, calculate a consistent estimator as the MLE for the parameter and calculate the pivotal quantity $Z$ based on the unknown parameter and its MLE. Thus, we have an objective and informative random sample from the pivotal quantities $Z=\left(z_{1}, z_{1}, \ldots, z_{n}\right)$ of size $n$, which constitute the sampling distribution for the pivotal $Z$.

4- Finally, based on the informative sample in step 3, we can use the AKDE for estimating $g(Z)$ at any given value for $Z$ and thus the confidence interval of the unknown parameter can be derived fiducially. 
Utilizing the above algorithm, the AKDE of the quantile $Z{ }_{p}$ of order $p$, for $Z$ can be derived as:

$$
\begin{aligned}
& \qquad\left(z_{p}\right)=\int_{-\infty}^{z} \hat{g}(z) d z=\frac{1}{n} \sum_{i=1-\infty}^{n} \int_{i}^{z} \frac{1}{h_{i}} K\left(\frac{z-z}{h_{i}}\right) d z=p . \\
& \text { Thus } \sum_{i=1}^{n} I\left(\frac{p{ }^{z} i}{h}\right)=n p \\
& \text { where } I(x)=\int_{-\infty}^{z} K(y) d y .
\end{aligned}
$$

For deriving the value of the quantile estimator $Z_{p}$, equation (6) can be solved recurrently as the limit to the sequence $\left\{\tilde{Z}_{1}, \tilde{Z}_{2}, \tilde{Z}_{3}, \ldots\right\}$, that defined by the formulas:

$$
\begin{gathered}
\tilde{Z}_{1}=\frac{1}{n} \sum_{i=1}^{n} Z_{i}, \\
\tilde{Z}_{r+1}=\tilde{Z}_{r}+C\left[n p-\sum_{i=1}^{n} I\left(\frac{\tilde{Z}_{r}-Z_{i}}{h_{i}}\right)\right], r=1,2,3, \ldots
\end{gathered}
$$

The convergence of (7) is guaranteed by the condition, $0<C<\frac{2 h_{i}}{n L_{1}}$, where $L_{1}=k(0)$, see Kulczycki (1999). 
For censored samples, we have to introduce another form to the kernel density estimation function which is the weighted kernel density estimation function and is defined as:

$$
\begin{aligned}
& \hat{f}(x)=\sum_{i=1}^{r} \frac{1}{h_{i} \alpha_{i}^{r}} K\left(\frac{x-x_{i}}{h_{i}}\right), \\
& \text { where } \alpha_{i}^{r}=\sum_{i=1}^{r} \frac{1}{r-m_{i}+1} .
\end{aligned}
$$

In this case, the kernel function can be taken as the truncated normal distribution which is defined as:

$K(x)=\left[\begin{array}{c}\left.\frac{1}{h_{i} \sqrt{2 \prod \Phi\left(\left(m_{i}+1\right) x_{i}\right)}} \exp -\frac{1}{2}\left(\frac{x-x_{i}}{h_{i}}\right)^{2}\right] \text { if } m_{i}=0, \quad i=1, \ldots, r-1, \quad m_{r}=n-r-1 \\ \frac{1}{h_{i} \sqrt{2 \prod} \Phi\left(m_{i} x_{i}\right)} \exp \left[-\frac{1}{2}\left(\frac{x-x_{i}}{h_{i}}\right)^{2}\right] \text { if } m_{i} \neq 0, \quad i=1, \ldots, r-1, \quad m_{r}=k-1\end{array}\right.$,

where $\Phi(z)=\frac{1}{\sqrt{2 \Pi}} \int_{-\infty}^{z} e^{-\frac{x^{2}}{2}} d x$, see Bordes (2004).

\subsection{Asymptotic Maximum Likelihood Estimation}

The MLE is a popular statistical method used for deriving the classical confidence interval for the distribution parameters. It provides statistically studies for the parameters and can be regarded as reference technique as in our study. For purpose of comparison, we obtain the confidence intervals for the parameters, thus the 
asymptotic variance-covariance matrix of the MLEs can be derived, which is the inversion of the Fisher information matrix whose elements are the negative of the expected values of the second order partial derivatives of the logarithm of the likelihood function.

The likelihood function based on the first n GOS for the generalized gamma distribution in (1) can be derived as:

$$
\begin{aligned}
& \ln L(\alpha, \beta, \lambda) \propto n \ln \alpha-n \alpha \lambda \ln \beta+(\alpha \lambda-1) \sum_{i=1}^{n} \ln \left(x_{i}\right)-\sum_{i=1}^{n}\left(x_{i} / \beta\right)^{\alpha} \\
& +\sum_{i=1}^{n} m_{i} \ln \left[\Gamma(\lambda)-I G\left(\lambda,\left(x_{i} / \beta\right)^{\alpha}\right)\right], \quad m_{n}=k-1 .
\end{aligned}
$$

The maximum likelihood estimators $\hat{\alpha}$ and $\hat{\beta}$ are the solutions to the system of equations obtained by equating to zero the first partial derivatives of the natural logarithm of the likelihood function with respect to $\alpha$ and $\beta$ when $\lambda$ is known. Thus, the ML estimators $\hat{\alpha}$ and $\hat{\beta}$ for $\alpha$ and $\beta$ respectively, can be obtained from the solution of the following normal equations:

$$
\begin{gathered}
\frac{\partial \ln L}{\alpha}=\frac{n}{\alpha}-n \lambda \ln \beta+\lambda \sum_{i=1}^{n} \ln x_{i}-\sum_{i=1}^{n}\left(x_{i} / \beta\right)^{\alpha} \ln \left(x_{i} / \beta\right) \\
-\sum^{*} \frac{\left(x_{i} / \beta\right)^{\alpha \lambda} \exp \left[-\left(x_{i} / \beta\right)^{\alpha}\right] \ln \left(x_{i} / \beta\right)}{\Gamma(\lambda)-I G\left(\lambda,\left(x_{i} / \beta\right)^{\alpha}\right)}=0,
\end{gathered}
$$

in expression (11) and later expressions, we use for convenience the summation notation:

$$
\Sigma^{*} g_{i}=\sum_{i=1}^{n} m_{i} g_{i}, \quad m_{n}=k-1
$$




$$
\frac{\partial \log L}{\beta}=-\frac{n \alpha \lambda}{\beta}+\frac{\alpha}{\beta} \sum_{i=1}^{n}\left(x_{i} / \beta\right)^{\alpha}+\frac{\alpha}{\beta} \sum^{*} \frac{\left(x_{i} / \beta\right)^{\alpha \lambda} \exp \left[\left(x_{i} / \beta\right)^{\alpha}\right]}{\Gamma(\lambda)-I G\left(\lambda,\left(x_{i} / \beta\right)^{\alpha}\right)}=0
$$

Equations (11) and (12) can't be solved analytically; statistical software can be used to solve these equations numerically. The Fisher information matrix $\boldsymbol{I}(\boldsymbol{\theta})$ can be constructed by differentiating (10) with respect to $\alpha$ and $\beta$ respectively when $\hat{\alpha}$ and $\hat{\beta}$ are known. Thus, the elements of $\boldsymbol{I}(\boldsymbol{\theta})$ have been derived as follows:

$$
\begin{aligned}
& I_{\alpha \alpha}=-\frac{\partial^{2} \log L}{\partial \alpha^{2}}=\frac{n}{\alpha^{2}}+\sum_{i=1}^{n}\left(x_{i} / \beta\right)^{\alpha}\left[\log \left(x_{i} / \beta\right)\right]^{2}+\sum^{*}\left[\frac{\left.\left(x_{i} / \beta\right)^{\alpha \lambda} \operatorname{expE}\left(x_{i} / \beta\right)^{\alpha}\right] \log \left(x_{i} / \beta\right)}{\Gamma(\lambda)-I G\left(\lambda,\left(x_{i} / \beta\right)^{\alpha}\right)}\right]^{2} \\
& +\sum \frac{\left.*\left(x_{i} / \beta\right)^{\alpha \lambda} \exp \left[-\left(x_{i} / \beta\right)^{\alpha}\right] \log \left(x_{i} / \beta\right)\right)^{2}\left[\lambda-\left(x_{i} / \beta\right)^{\alpha}\right]}{\Gamma(\lambda)-I G\left(\lambda,\left(x_{i} / \beta\right)^{\alpha}\right)}, \\
& I_{\beta \beta}=-\frac{\partial^{2} L n L}{\partial \beta^{2}}=-\frac{n \alpha \lambda}{\beta^{2}}+\frac{\alpha(\alpha+1)}{\beta^{2}} \sum_{i=1}^{n}\left(x_{i} / \beta\right)^{\alpha}+\frac{\alpha}{\beta^{2}} \Sigma^{*}\left[\frac{\left(x_{i} / \beta\right)^{\alpha \lambda} \exp \left[-\left(x_{i} / \beta\right)^{\alpha}\right]}{\Gamma(\lambda)-I G\left(\lambda,\left(x_{i} / \beta\right)^{\alpha}\right)}\right] \\
& -\frac{\alpha^{2}}{\beta^{2}} \Sigma^{*}\left(\frac{\left(x_{i} / \beta\right)^{\alpha \lambda} \exp \left[-\left(x_{i} / \beta\right)^{\alpha}\right]\left[-\lambda+\left(x_{i} / \beta\right)^{\alpha}\right]}{\Gamma(\lambda)-I G\left(\lambda,\left(x_{i} / \beta\right)^{\alpha}\right)}-\left[\frac{\left(x_{i} / \beta\right)^{\alpha \lambda} \exp \left[-\left(x_{i} / \beta\right)^{\alpha}\right]}{\Gamma(\lambda)-I G\left(\lambda,\left(x_{i} / \beta\right)^{\alpha}\right)}\right]^{2}\right),
\end{aligned}
$$

and

$$
I_{\alpha \beta}=-\frac{\partial^{2} \log L}{\partial \alpha \partial \beta}=\frac{n \lambda}{\beta}-\frac{\alpha}{\beta} \sum_{i=1}^{n}\left(x_{i} / \beta\right)^{\alpha} \ln \left(x_{i} / \beta\right)-\frac{1}{\beta} \sum_{i=1}^{n}\left(x_{i} / \beta\right)^{\alpha}
$$




$$
\begin{gathered}
-\sum \frac{\frac{1}{\beta}\left(x_{i} / \beta\right)^{\alpha \lambda} \exp \left(-\left(x_{i} / \beta\right)^{\alpha}\right)\left[1-\alpha\left(x_{i} / \beta\right)^{\alpha} \log \left(x_{i} / \beta\right)+\alpha \lambda \log \left(x_{i} / \beta\right)\right]}{\Gamma(\lambda)-I G\left(\lambda,\left(x_{i} / \beta\right)^{\alpha}\right)} \\
\left.\left.\left.-\sum * \frac{\frac{\alpha}{\beta}\left(\left(x_{i} / \beta\right)^{\alpha \lambda} \exp \left(-\left(x_{i} / \beta\right)^{\alpha}\right)\right)^{2} \log \left(x_{i} / \beta\right)}{\left(\Gamma(\lambda)-I G\left(\lambda,\left(x_{i} / \beta\right)\right.\right.}\right)^{\alpha}\right)\right)^{2}
\end{gathered}
$$

Therefore, the asymptotic Fisher's information matrix can be written as:

$$
F=\left[\begin{array}{ll}
I_{\alpha \alpha} & I_{\alpha \beta} \\
I_{\beta \alpha} & I_{\beta \beta}
\end{array}\right]_{(\alpha, \beta)=(\hat{\alpha}, \hat{\beta})}
$$

In relation to the asymptotic variance-covariance matrix of the ML estimators of the parameters, it can be approximated numerically by inverting the above Fisher's information matrix F. The approximate $100(1-\gamma) \%$ two- sided confidence intervals for $\alpha$ and $\beta$ can be, respectively, obtained as

$$
\hat{\alpha} \pm Z_{\gamma / 2} \hat{\sigma}(\hat{\alpha}) \text { and } \hat{\beta} \pm Z_{\gamma / 2} \hat{\sigma}(\hat{\beta})
$$

where $Z_{\gamma / 2}$ is the upper $(\gamma / 2)^{t h}$ percentile of a standard distribution, and $\hat{\sigma}(\hat{\alpha})$, $\hat{\sigma}(\hat{\beta})$ are, respectively, the standard deviations of the ML estimators of the parameters $\alpha$ and $\beta$.

\section{Simulation Study and Comparisons}

To assess the performance of the confidence intervals based on the kernel approach comparing to those based on the asymptotic maximum likelihood 
estimation approach, Monte Carlo simulations are carried out, in terms of the following criteria:

i) Covering percentage (CP), which is defined as the fraction of times the confidence interval covers the true value of the parameter in repeated sampling.

ii) The mean length of intervals (MLI).

iii) The standard error of the covering percentage (SDE), which is defined for the nominal $\quad$ level $(1-\alpha) 100 \%$ by $\quad \operatorname{SDE}(\hat{\alpha})=\sqrt{\frac{\hat{\alpha}(1-\hat{\alpha})}{M}}$ where $(1-\hat{\alpha}) 100 \%$ denote the corresponding Monte Carlo estimate and $\mathrm{M}$ is the number of Monte Carlo trials. Thus, for the nominal level 95\% and 1000 simulation trails, say, the standard error of the covering percentage is 0.0049 , which is approximately $\pm 1 \%$. Therefore, we say the procedure is adequate if the SDE is within $\pm 2 \%$ error for the nominal level 95\%.

The results, based on 1000 Monte Carlo simulations are given for samples of sizes n $=20,40$ and 80 , which have been generated for true values of the scale parameter $\beta$

$=2,3$, shape parameter $\alpha=1,2$ and $\lambda=1,2$ based on the complete, the type-II censored and type-II progressive censored samples with binomial random removals at $\mathrm{P}=0.5$ and uncensored levels $\mathrm{r}$ equals [n/2] and [3n/4]. From the simulation study, we summarized some of the interesting features in the following points:

1- The results in Tables (2-3) indicated that, as the sample size increases, the values of MLIs getting decrease and the values of CPs increase, while the values of $S D E$ s decrease for all values of $\alpha$, for the two approaches, based on the complete, type-II censored and type-II progressive censored samples.

2- The mean length of intervals for the parameter $\alpha$ increase as the shape parameter increases as would be expected. On the contrary, for the parameter $\beta$, the values of MLIs decrease as the shape parameter $\alpha$ increases for the complete, type-II censored and type-II progressive censored samples, based on the AML approach. However, the values of MLIs, CPs and SDEs, based on the kernel approach, are fixed for increasing the values of $\alpha$ based on the complete, typeII censored and type-II progressive censored samples. 
3- As the true value $\lambda$ increases the values of MLIs decrease, and the CPs mostly increase and the values of SDEs decrease based on the complete, type-II censored and type-II progressive censored samples.

4- The kernel approach is conservative for estimating the parameters $\alpha$ and $\beta$ because the covering percentages are much greater than the nominal level for those based on the classical inference for all sample sizes. On the contrary, the classical approach is anti-conservative for estimating $\alpha$ and almost conservative for $\beta$, when the sample size is greater than 20 .

5- It is worthwhile to note that, the mean length and the values of SDEs based on the type-II progressive censored samples are less than those based on type-II censored samples. Moreover, the values of CPs for type-II progressive censored samples are greater than those based on type-II censored samples.

6- Finally, both the two procedures are adequate because the values of SDEs are less than \pm 2 for the nominal level $95 \%$.

\section{An illustrative Example}

Consider the results of tests, the endurance of deep groove ball bearings. The data are quoted from Lawless (1980) consist of a complete sample of size $n=23$, that represent the results of the test, in millions of revolutions before failures are: 17.88, 28.92, 33.00, 41.52, 41.12, 45.60, 48.48, 51.84, 51.96, 54.12, 55.56, 67.80, 68.64, 68.64, 68.88, 84.12, 93.12, 98.64, 105.12, 105.84, 127.92, 128.04, 173.40.

Thus, for the purpose of comparison, the 95\% CIs for the parameters $\alpha$ and $\beta$ are derived based on the two approaches, based on complete, type-II censored and type-II progressive censored samples with binomial removals at $\mathrm{P}=0.5$ and uncensored levels $\mathrm{r}$ equal to [n/2] and [3n/4] for $\lambda=1,2$.

The results in Table 1 have been indicated that the length of intervals for $\alpha$ and $\beta$ based on the kernel approach are shorter than those based on the classical inference. Furthermore, clearly, the length of intervals decrease when the true value of $\lambda$ increases. Finally, it is worthwhile to note that, the length of intervals based on the type-II progressive censored samples are smaller than those based on type-II censored samples, which ensure the simulation results. 
M. Ahsanullah, M. Maswadah and Seham Ali M.

Table 1: The Lower (LL) and the Upper limits (UL), Maximum likelihood estimates (MLE), and the lengths of the 95\% confidence intervals (CI) for the parameters for $\alpha$ and $\beta$ using the kernel and AMLs approaches based on GOS based on the data.

\begin{tabular}{|c|c|c|c|c|c|c|c|}
\hline \multicolumn{6}{|c|}{ Approaches } & Kernel & AMLs \\
\hline \multicolumn{6}{|c|}{$\overline{C I}$} & $95 \%$ & $95 \%$ \\
\hline Par. & $S$ & $n$ & $r$ & $\lambda$ & $M L E$ & UL & UL \\
\hline \multirow{10}{*}{$\alpha$} & \multirow{6}{*}{$\begin{array}{l}\text { Type-II } \\
\text { censored } \\
\text { sample }\end{array}$} & \multirow[t]{6}{*}{23} & \multirow[t]{2}{*}{11} & 1 & 4.8410 & 1.3999 (4.0361) 5.4360 & 1.6503 (4.8486) 5.7799 \\
\hline & & & & 2 & 2.9515 & 0.8424 (2.3094) 3.1518 & 1.5281 (2.8469) 4.3750 \\
\hline & & & \multirow[t]{2}{*}{17} & 1 & 3.1493 & 0.8195 (1.8297) 2.6492 & 1.3622 (2.3036) 3.2237 \\
\hline & & & & 2 & 2.0903 & 0.5672 (1.1459) 1.7131 & 1.3448 (1.4909) 2.8357 \\
\hline & & & \multirow[t]{2}{*}{23} & 1 & 2.1021 & 1.3975 (1.1594) 2.5569 & 1.4578 (1.2885) 2.7463 \\
\hline & & & & 2 & 1.4378 & 1.0002 (0.7596) 1.7598 & 1.0075 (0.8607) 1.8682 \\
\hline & \multirow{4}{*}{$\begin{array}{l}\text { Type-II } \\
\text { progressive } \\
\text { censored } \\
\text { sample }\end{array}$} & & 11 & 1 & 3.7153 & 1.1876 (4.0274) 5.2150 & 1.6503 (4.1296) 5.7799 \\
\hline & & & & 2 & 2.1852 & 2.2245 (2.0860) 4.3105 & 1.0276 (2.3151) 3.3427 \\
\hline & & & 17 & 1 & 2.2926 & 1.1725 (2.6022) 3.7747 & 1.3622 (1.8615) 3.2237 \\
\hline & & & & 2 & 1.4862 & 1.5660 (1.3660) 2.9320 & 0.9077 (1.1570) 2.0647 \\
\hline \multirow{10}{*}{$\beta$} & \multirow{6}{*}{$\begin{array}{l}\text { Type-II } \\
\text { censored } \\
\text { sample }\end{array}$} & \multirow[t]{6}{*}{23} & \multirow[t]{2}{*}{11} & 1 & 62.9426 & 60.1484 (38.7903) 98.9387 & 51.4351 (23.0159) 74.4510 \\
\hline & & & & 2 & 45.3160 & 36.2246 (24.0632) 60.2878 & 36.7370 (17.1580) 53.8950 \\
\hline & & & \multirow[t]{2}{*}{17} & 1 & 79.4376 & 71.5374 (57.5415) 129.0789 & 62.9614 (32.9544) 95.9158 \\
\hline & & & & 2 & 47.1196 & 32.3249 (23.0072) 55.3321 & 34.2310 (25.7772) 60.0082 \\
\hline & & & \multirow[t]{2}{*}{23} & 1 & 81.8783 & 59.9192 (52.4844) 112.4036 & 65.0215 (33.7137) 98.7352 \\
\hline & & & & 2 & 46.9838 & 29.6682 (32.0680) 61.7362 & 33.8571 (26.2534) 60.1105 \\
\hline & \multirow{4}{*}{$\begin{array}{l}\text { Type-II } \\
\text { progressive } \\
\text { censored } \\
\text { sample }\end{array}$} & \multirow[t]{4}{*}{23} & \multirow[t]{2}{*}{11} & 1 & 46.9182 & 45.2633 (13.7753) 59.0386 & 41.0428 (11.7507) 52.7935 \\
\hline & & & & 2 & 35.8730 & 31.8807 (13.5430) 45.4237 & 28.8399 (14.0662) 42.9061 \\
\hline & & & 17 & 1 & 60.6451 & 58.1276 (28.7593) 86.8869 & 51.0865 (19.1172) 70.2037 \\
\hline & & & & 2 & 41.4204 & 33.7310 (17.4136) 51.1446 & $32.3111(18.2186) 50.5297$ \\
\hline
\end{tabular}


Table 2. The (MLIs), (CPs) and (SDEs) for the kernel and the AMLs approaches when the nominal level is $95 \%$ for the parameter $\boldsymbol{\alpha}$ with ( $\boldsymbol{\beta}=2, \boldsymbol{3}$ ) for the censored levels ( $50 \%$ and $75 \%$ ).

\begin{tabular}{|c|c|c|c|c|c|c|c|c|c|c|c|c|}
\hline \multicolumn{3}{|c|}{ App. } & \multicolumn{5}{|c|}{ Kernel } & \multicolumn{5}{|c|}{ AMLs } \\
\hline & & & \multicolumn{5}{|c|}{ MLI,$\alpha$} & \multicolumn{5}{|c|}{ MLI,$\alpha$} \\
\hline$n$ & $\bar{r}$ & $\bar{\lambda}$ & 1.0 & 2.0 & 3.0 & $C P$ & SDE & 1.0 & 2.0 & 3.0 & $\overline{C P}$ & SDE \\
\hline \multicolumn{13}{|c|}{ Complete and type-II censored samples } \\
\hline \multirow[t]{6}{*}{20} & 10 & 1 & 1.7687 & 3.5373 & 5.3060 & 0.945 & 0.0072 & 1.3914 & 2.7828 & 4.1742 & 0.960 & 0.0062 \\
\hline & 15 & & 1.1243 & 2.2486 & 3.3728 & 0.944 & 0.0073 & 0.9890 & 1.9780 & 2.9669 & 0.958 & 0.0063 \\
\hline & 20 & & 0.8344 & 1.6688 & 2.5032 & 0.943 & 0.0073 & 0.7415 & 1.4831 & 2.2246 & 0.958 & 0.0063 \\
\hline & 10 & 2 & 1.7914 & 3.5816 & 5.3743 & 0.946 & 0.0071 & 1.2913 & 2.5826 & 3.8738 & 0.961 & 0.0061 \\
\hline & 15 & & 1.1173 & 2.2345 & 3.3518 & 0.945 & 0.0072 & 0.9356 & 1.8712 & 2.8067 & 0.947 & 0.0071 \\
\hline & 20 & & 0.7889 & 1.5779 & 2.3668 & 0.932 & 0.008 & 0.7118 & 1.4236 & 2.1354 & 0.931 & 0.0080 \\
\hline \multirow[t]{6}{*}{40} & 20 & 1 & 0.9884 & 1.9768 & 2.9653 & 0.938 & 0.0076 & 0.8964 & 1.7928 & 2.6892 & 0.950 & 0.0069 \\
\hline & 30 & & 0.8203 & 1.6405 & 2.4608 & 0.953 & 0.0067 & 0.6608 & 1.3217 & 1.9825 & 0.953 & 0.0067 \\
\hline & 40 & & 0.5600 & 1.1200 & 1.6800 & 0.956 & 0.0065 & 0.5032 & 1.0063 & 1.5095 & 0.955 & 0.0066 \\
\hline & 20 & 2 & 0.9179 & 1.8358 & 2.7537 & 0.948 & 0.0070 & 0.8453 & 1.6905 & 2.5358 & 0.951 & 0.0068 \\
\hline & 30 & & 0.7299 & 1.4598 & 2.1897 & 0.951 & 0.0068 & 0.6282 & 1.2563 & 1.8845 & 0.949 & 0.0070 \\
\hline & 40 & & 0.5365 & 1.0730 & 1.6095 & 0.942 & 0.0074 & 0.4832 & 0.9665 & 1.4497 & 0.939 & 0.0076 \\
\hline \multirow[t]{6}{*}{80} & 40 & 1 & 0.7057 & 1.4114 & 2.1171 & 0.952 & 0.0068 & 0.6007 & 1.2015 & 1.8022 & 0.951 & 0.0068 \\
\hline & 60 & & 0.5128 & 1.0255 & 1.5383 & 0.955 & 0.0066 & 0.4561 & 0.9122 & 1.3682 & 0.952 & 0.0068 \\
\hline & 80 & & 0.3831 & 0.7662 & 1.1493 & 0.957 & 0.0064 & 0.3486 & 0.6973 & 1.0459 & 0.955 & 0.0066 \\
\hline & 40 & 2 & 0.6828 & 1.3657 & 2.0485 & 0.958 & 0.0063 & 0.5701 & 1.1402 & 1.7103 & 0.962 & 0.0060 \\
\hline & 60 & & 0.4982 & 0.9964 & 1.4946 & 0.955 & 0.0066 & 0.4307 & 0.8615 & 1.2922 & 0.955 & 0.0066 \\
\hline & 80 & & 0.3667 & 0.7333 & 1.1000 & 0.951 & 0.0068 & 0.3334 & 0.6668 & 1.0002 & 0.945 & 0.0072 \\
\hline
\end{tabular}


M. Ahsanullah, M. Maswadah and Seham Ali M.

Table2. (Continued)

\begin{tabular}{|c|c|c|c|c|c|c|c|c|c|c|c|c|}
\hline \multicolumn{3}{|c|}{ App. } & \multicolumn{4}{|c|}{ Kernel } & \multicolumn{6}{|c|}{ AMLs } \\
\hline & & & \multicolumn{4}{|c|}{ MLI , $\alpha$} & \multicolumn{6}{|c|}{ MLI , $\alpha$} \\
\hline $\bar{n}$ & $\bar{r}$ & $\bar{\lambda}$ & 1.0 & 2.0 & 3.0 & $\overline{C P}$ & SDE & 1.0 & 2.0 & 3.0 & $\overline{C P}$ & $\overline{\mathrm{SDE}}$ \\
\hline \multicolumn{13}{|c|}{ Type-II progressive censored samples } \\
\hline \multirow[t]{4}{*}{20} & 10 & 1 & 1.4774 & 2.9549 & 4.4323 & 0.972 & 0.0052 & 1.1495 & 2.2990 & 3.4485 & 0.940 & 0.0075 \\
\hline & 15 & & 1.0995 & 2.1991 & 3.2986 & 0.960 & 0.0062 & 0.8865 & 1.7725 & 2.6594 & 0.959 & 0.0063 \\
\hline & 10 & 2 & 1.2121 & 2.4242 & 3.6364 & 0.958 & 0.0063 & 1.1073 & 2.2145 & 3.3218 & 0.951 & 0.0068 \\
\hline & 15 & & 1.0708 & 2.1416 & 3.2124 & 0.958 & 0.0063 & 0.8482 & 1.6965 & 2.5447 & 0.952 & 0.0068 \\
\hline \multirow[t]{4}{*}{40} & 20 & 1 & 1.0580 & 2.1159 & 3.1739 & 0.978 & 0.0046 & 0.7396 & 1.4792 & 2.2188 & 0.949 & 0.0070 \\
\hline & 30 & & 0.7852 & 1.5703 & 2.3555 & 0.983 & 0.0041 & 0.5915 & 1.1831 & 1.7746 & 0.944 & 0.0073 \\
\hline & 20 & 2 & 0.8418 & 1.6835 & 2.5253 & 0.971 & 0.0053 & 0.7087 & 1.4174 & 2.1261 & 0.939 & 0.0076 \\
\hline & 30 & & 0.7615 & 1.5231 & 2.2846 & 0.980 & 0.0044 & 0.5668 & 1.1336 & 1.7004 & 0.935 & 0.0078 \\
\hline \multirow[t]{4}{*}{80} & 40 & 1 & 0.7147 & 1.4295 & 2.1442 & 0.959 & 0.0063 & 0.5053 & 1.0105 & 1.5158 & 0.950 & 0.0069 \\
\hline & 60 & & 0.6544 & 1.3088 & 1.9632 & 0.975 & 0.0049 & 0.4036 & 0.8073 & 1.2110 & 0.962 & 0.0060 \\
\hline & 40 & 2 & 0.6970 & 1.3940 & 2.0911 & 0.976 & 0.0048 & 0.4785 & 0.9570 & 1.4355 & 0.959 & 0.0063 \\
\hline & 60 & & 0.6479 & 1.2958 & 1.9437 & 0.971 & 0.0053 & 0.3877 & 0.7754 & 1.1631 & 0.955 & 0.0066 \\
\hline
\end{tabular}

\section{Conclusions}

The kernel estimation technique constitutes a strong basis for statistical inference, and it has a number of benefits relative to the usual classical procedure. First, it is easy to be implemented, and it doesn't need tedious work as the classical inference. Second, it can perform quite well even when the number of bootstraps is extremely small up to 20 replications. Finally, it is uniquely determined based on the information content in the pivotal quantities and thus, we can consider it as an alternative and reliable technique for estimation stronger than the classical inference. 
Table 3. The (MLIs), (CPs) and (SDEs) for the kernel and the AMLs approaches when the nominal level is 95\% for the parameter $\boldsymbol{\beta}$ based on the complete, type-II censored and type-II progressive censored samples with censored levels ( 50\% and 75\%).

\begin{tabular}{|c|c|c|c|c|c|c|c|c|c|c|}
\hline \multicolumn{5}{|c|}{ App. } & \multicolumn{3}{|c|}{ Kernel } & \multicolumn{3}{|c|}{ AMLs } \\
\hline$n$ & $\bar{r}$ & $\lambda$ & $\beta$ & $\alpha$ & MLI & $\mathrm{CP}$ & SDE & MLI & $\mathrm{CP}$ & $\mathrm{SDE}$ \\
\hline \multicolumn{11}{|c|}{ Complete and type-II censored samples } \\
\hline \multirow[t]{12}{*}{20} & 10 & 1 & 2 & 1 & 6.7292 & 0.841 & 0.0116 & 2.5376 & 0.797 & 0.0127 \\
\hline & & & & 2 & 3.5565 & 0.841 & 0.0116 & 1.2487 & 0.827 & 0.0120 \\
\hline & & & 3 & 1 & 11.0938 & 0.841 & 0.0116 & 3.8064 & 0.797 & 0.0127 \\
\hline & & & & 2 & 5.3348 & 0.841 & 0.0116 & 1.8730 & 0.827 & 0.0120 \\
\hline & 15 & & 2 & 1 & 3.2168 & 0.947 & 0.0071 & 1.8998 & 0.893 & 0.0098 \\
\hline & & & & 2 & 1.4051 & 0.947 & 0.0071 & 0.9473 & 0.908 & 0.0091 \\
\hline & & & 3 & 1 & 4.8251 & 0.947 & 0.0071 & 2.8497 & 0.893 & 0.0098 \\
\hline & & & & 2 & 2.1076 & 0.947 & 0.0071 & 1.4209 & 0.908 & 0.0091 \\
\hline & 20 & & 2 & 1 & 2.5416 & 0.957 & 0.0064 & 1.7691 & 0.925 & 0.0083 \\
\hline & & & & 2 & 1.1738 & 0.957 & 0.0064 & 0.8816 & 0.925 & 0.0083 \\
\hline & & & 3 & 1 & 3.8124 & 0.957 & 0.0064 & 2.6537 & 0.925 & 0.0083 \\
\hline & & & & 2 & 1.7607 & 0.957 & 0.0064 & 1.3223 & 0.925 & 0.0083 \\
\hline \multirow[t]{12}{*}{20} & 10 & 2 & 2 & 1 & 2.0419 & 0.933 & 0.0079 & 1.6267 & 0.894 & 0.0097 \\
\hline & & & & 2 & 1.0072 & 0.933 & 0.0079 & 0.8080 & 0.891 & 0.0099 \\
\hline & & & 3 & 1 & 3.0628 & 0.933 & 0.0079 & 2.4401 & 0.894 & 0.0097 \\
\hline & & & & 2 & 1.5108 & 0.933 & 0.0079 & 1.2120 & 0.891 & 0.0099 \\
\hline & 15 & & 2 & 1 & 1.9159 & 0.933 & 0.0079 & 1.6751 & 0.918 & 0.0087 \\
\hline & & & & 2 & 0.9302 & 0.933 & 0.0079 & 0.8289 & 0.914 & 0.0089 \\
\hline & & & 3 & 1 & 2.8738 & 0.933 & 0.0079 & 2.5127 & 0.918 & 0.0087 \\
\hline & & & & 2 & 1.3953 & 0.933 & 0.0079 & 1.2434 & 0.914 & 0.0089 \\
\hline & 20 & & 2 & 1 & 1.8545 & 0.914 & 0.0089 & 1.6911 & 0.926 & 0.0083 \\
\hline & & & & 2 & 0.9292 & 0.914 & 0.0089 & 0.8340 & 0.923 & 0.0084 \\
\hline & & & 3 & 1 & 2.7817 & 0.914 & 0.0089 & 2.5367 & 0.926 & 0.0083 \\
\hline & & & & 2 & 1.3939 & 0.914 & 0.0089 & 1.2511 & 0.923 & 0.0084 \\
\hline
\end{tabular}


M. Ahsanullah, M. Maswadah and Seham Ali M.

Table3. (Continued)

\begin{tabular}{|c|c|c|c|c|c|c|c|c|c|c|}
\hline \multicolumn{11}{|c|}{ Complete and type-II censored samples } \\
\hline \multirow[t]{12}{*}{40} & 20 & 1 & 2 & 1 & 4.1061 & 0.961 & 0.0061 & 1.8354 & 0.876 & 0.0104 \\
\hline & & & & 2 & 1.6157 & 0.961 & 0.0061 & 0.9176 & 0.897 & 0.0096 \\
\hline & & & 3 & 1 & 6.1592 & 0.961 & 0.0061 & 2.7531 & 0.876 & 0.0104 \\
\hline & & & & 2 & 2.4236 & 0.961 & 0.0061 & 1.3764 & 0.897 & 0.0096 \\
\hline & 30 & & 2 & 1 & 1.6999 & 0.967 & 0.0056 & 1.3803 & 0.940 & 0.0075 \\
\hline & & & & 2 & 0.8184 & 0.967 & 0.0056 & 0.6909 & 0.944 & 0.0073 \\
\hline & & & 3 & 1 & 2.5499 & 0.967 & 0.0056 & 2.0704 & 0.940 & 0.0075 \\
\hline & & & & 2 & 1.2276 & 0.967 & 0.0056 & 1.0364 & 0.944 & 0.0073 \\
\hline & 40 & & 2 & 1 & 1.6590 & 0.974 & 0.0050 & 1.2692 & 0.954 & 0.0066 \\
\hline & & & & 2 & 0.8032 & 0.974 & 0.0050 & 0.6351 & 0.946 & 0.0071 \\
\hline & & & 3 & 1 & 2.4885 & 0.974 & 0.0050 & 1.9038 & 0.954 & 0.0066 \\
\hline & & & & 2 & 1.2048 & 0.974 & 0.0050 & 0.9527 & 0.946 & 0.0071 \\
\hline \multirow[t]{12}{*}{40} & 20 & 2 & 2 & 1 & 1.4617 & 0.947 & 0.0071 & 1.1938 & 0.914 & 0.0089 \\
\hline & & & & 2 & 0.7161 & 0.947 & 0.0071 & 0.5938 & 0.914 & 0.0089 \\
\hline & & & 3 & 1 & 2.1926 & 0.947 & 0.0071 & 1.7907 & 0.914 & 0.0089 \\
\hline & & & & 2 & 1.0742 & 0.947 & 0.0071 & 0.8906 & 0.914 & 0.0089 \\
\hline & 30 & & 2 & 1 & 1.3103 & 0.951 & 0.0068 & 1.2116 & 0.930 & 0.0081 \\
\hline & & & & 2 & 0.6598 & 0.951 & 0.0068 & 0.6012 & 0.926 & 0.0083 \\
\hline & & & 3 & 1 & 1.9654 & 0.951 & 0.0068 & 1.8175 & 0.930 & 0.0081 \\
\hline & & & & 2 & 0.9896 & 0.951 & 0.0068 & 0.9018 & 0.926 & 0.0083 \\
\hline & 40 & & 2 & 1 & 1.4950 & 0.957 & 0.0064 & 1.2114 & 0.941 & 0.0075 \\
\hline & & & & 2 & 0.7377 & 0.957 & 0.0064 & 0.6004 & 0.937 & 0.0077 \\
\hline & & & 3 & 1 & 2.2425 & 0.957 & 0.0064 & 1.8171 & 0.941 & 0.0075 \\
\hline & & & & 2 & 1.1065 & 0.957 & 0.0064 & 0.9007 & 0.937 & 0.0077 \\
\hline
\end{tabular}


Kernel Inference on the Generalized Gamma Distribution

\begin{tabular}{|c|c|c|c|c|c|c|c|c|c|c|}
\hline \multicolumn{11}{|c|}{ Type-II progressive censored samples } \\
\hline \multirow[t]{16}{*}{20} & \multirow[t]{4}{*}{10} & \multirow[t]{8}{*}{1} & \multirow[t]{2}{*}{2} & 1 & 3.7357 & 0.945 & 0.0072 & 2.3476 & 0.883 & 0.0102 \\
\hline & & & & 2 & 1.6194 & 0.945 & 0.0072 & 1.1793 & 0.896 & 0.0097 \\
\hline & & & \multirow[t]{2}{*}{3} & 1 & 5.6035 & 0.945 & 0.0072 & 3.5214 & 0.883 & 0.0102 \\
\hline & & & & 2 & 2.4291 & 0.945 & 0.0072 & 1.7689 & 0.896 & 0.0097 \\
\hline & \multirow[t]{4}{*}{15} & & \multirow[t]{2}{*}{2} & 1 & 2.5122 & 0.958 & 0.0063 & 1.9795 & 0.893 & 0.0098 \\
\hline & & & & 2 & 1.2250 & 0.958 & 0.0063 & 0.9894 & 0.904 & 0.0093 \\
\hline & & & \multirow[t]{2}{*}{3} & 1 & 3.7683 & 0.958 & 0.0063 & 2.9692 & 0.893 & 0.0098 \\
\hline & & & & 2 & 1.8375 & 0.958 & 0.0063 & 1.4842 & 0.904 & 0.0093 \\
\hline & \multirow{4}{*}{10} & \multirow[t]{8}{*}{2} & \multirow[t]{2}{*}{2} & 1 & 2.9915 & 0.917 & 0.0087 & 2.3464 & 0.903 & 0.0094 \\
\hline & & & & 2 & 1.3663 & 0.917 & 0.0087 & 1.1332 & 0.892 & 0.0098 \\
\hline & & & \multirow[t]{2}{*}{3} & 1 & 4.4872 & 0.917 & 0.0087 & 3.5196 & 0.903 & 0.0094 \\
\hline & & & & 2 & 2.0495 & 0.917 & 0.0087 & 1.6997 & 0.892 & 0.0098 \\
\hline & \multirow[t]{4}{*}{15} & & \multirow[t]{2}{*}{2} & 1 & 1.9672 & 0.945 & 0.0055 & 1.9515 & 0.933 & 0.0079 \\
\hline & & & & 2 & 1.0085 & 0.945 & 0.0055 & 0.9511 & 0.927 & 0.0082 \\
\hline & & & \multirow[t]{2}{*}{3} & 1 & 2.9508 & 0.945 & 0.0055 & 2.9273 & 0.933 & 0.0079 \\
\hline & & & & 2 & 1.5127 & 0.945 & 0.0055 & 1.4266 & 0.927 & 0.0082 \\
\hline \multirow[t]{16}{*}{40} & \multirow[t]{4}{*}{20} & \multirow[t]{8}{*}{$\overline{1}$} & \multirow[t]{2}{*}{2} & 1 & 2.2367 & 0.975 & 0.0049 & 1.7564 & 0.912 & 0.0090 \\
\hline & & & & 2 & 1.1289 & 0.975 & 0.0049 & 0.8785 & 0.919 & 0.0086 \\
\hline & & & \multirow[t]{2}{*}{3} & 1 & 3.3550 & 0.975 & 0.0049 & 2.6347 & 0.912 & 0.0090 \\
\hline & & & & 2 & 1.6934 & 0.975 & 0.0049 & 1.3177 & 0.919 & 0.0086 \\
\hline & \multirow[t]{4}{*}{30} & & \multirow[t]{2}{*}{2} & 1 & 1.5854 & 0.954 & 0.0066 & 1.4479 & 0.923 & 0.0084 \\
\hline & & & & 2 & 0.7887 & 0.954 & 0.0066 & 0.7249 & 0.925 & 0.0083 \\
\hline & & & \multirow[t]{2}{*}{3} & 1 & 2.3780 & 0.954 & 0.0066 & 2.1718 & 0.923 & 0.0084 \\
\hline & & & & 2 & 1.1831 & 0.954 & 0.0066 & 1.0873 & 0.925 & 0.0083 \\
\hline & \multirow[t]{4}{*}{20} & 2 & \multirow[t]{2}{*}{2} & 1 & 1.9092 & 0.964 & 0.0059 & 1.7028 & 0.926 & 0.0083 \\
\hline & & & & 2 & 0.9932 & 0.964 & 0.0059 & 0.8388 & 0.928 & 0.0082 \\
\hline & & & \multirow[t]{2}{*}{3} & 1 & 2.8637 & 0.964 & 0.0059 & 2.5542 & 0.926 & 0.0083 \\
\hline & & & & 2 & 1.4898 & 0.964 & 0.0059 & 1.2582 & 0.928 & 0.0082 \\
\hline & 30 & & 2 & 1 & 1.5262 & 0.955 & 0.0066 & 1.3959 & 0.928 & 0.0082 \\
\hline & & & & 2 & 0.7581 & 0.955 & 0.0066 & 0.6892 & 0.932 & 0.0080 \\
\hline & & & 3 & 1 & 2.2893 & 0.955 & 0.0066 & 2.0939 & 0.928 & 0.0082 \\
\hline & & & & 2 & 1.1371 & 0.955 & 0.0066 & 1.0338 & 0.932 & 0.0080 \\
\hline
\end{tabular}




\section{References}

1. E. Stacy, A generalization of the gamma distribution. Annals of Mathematical Statistics, (1962) 1187-1192.

2. E. Stacy and G. Mihram, Parameter estimation for a generalized gamma distribution. Technometrics. 7(1965) 349-358.

3. V. B. Parr and J. T. Webster, A Method for Discriminating Between Failure Density Functions Used in Reliability Predictions. Technometrics. 7(1965) 1-10.

4. H. L. Harter, Maximum likelihood estimation of the parameters of a four parameters generalized gamma population from complete and censored samples. Technometrics. 9(1967) 159-5.

5. H. Hager and L. Bain, Inferential procedures for the generalized gamma distribution. Journal of the American Statistical Association, 65(1970) 16011609.

6. R. Prentice, A log gamma model and its maximum likelihood estimation. Biometrika. 61(1974), 539-544.

7. J. Lawless, Inference on the generalized gamma and log gamma distributions. Technometrics, 22(1980), 409-419.

8. T. Di Ciccio, Approximate inference for the generalized gamma distribution. Technometrics, (1987) 33-40.

9. D. R. Wingo, Computing maximum-likelihood parameter estimates of the generalized gamma distribution by numerical root isolation. IEEE Transactions on Reliability, 36(1987) 586-590

10. J. Y. Wong, Readily obtaining the maximum-likelihood estimates of the 3parameter generalized gamma-distribution of Stacy and Mirham. Microelectronics and Reliability, 33(1993) 2243-2251.

11. A. C. Cohen and B. J. Whitten, Parameter Estimation in Reliability and Life Span Models. (Dekker, New York, 1988).

12. M. S. Maswadah, Two approaches based on the structural model to inference on the generalized Gamma parameters. Egyptian Statist. J., 33(1)(1989) 110-129.

13. M. S. Maswadah, Structural inference on the generalized Gamma distribution based on type-II progressively censored sample. J. Austral. Math. Soc. Ser. A, 50(1)(1991) 15-22.

14. T. Hwang and P. Huang, On new moment estimation of parameters of the Generalized Gamma distribution and its characterization. Taiwanese journal of Mathematics, 10(4)(2006) 1083-1093. 
15. A. Dadpay, E. Soofi and R. Soyer, Information measures for generalized gamma family. Journal of Econometrics, 56(2007) 568-585.

16. O. Gomes, C. Combes and A. Dussauchoy, (2008). Parameter estimation of the generalized gamma distribution. Mathematics and Computers in Simulation,79(2008) 955-963.

17. C. Geng and L. Yuhiong, A Note on the Maximum Likelihood Estimation for the Generalized Gamma Distribution Parameters under Progressive Type-II Censoring. International Journal of Intelligent Technology and Applied Statistics, 2(2)(2009) , PP. 57-64.

18. B. Mukherjee, A. Gupta and S. K. Upadhyay, A Bayesian study for the comparison of generalized gamma model with its components. Sankhya B, 72(2011), 154-174.

19. U. Kamps, A concept of generalized order statistics. (Germany: B. G. Teubner Stuttgart, 1995).

20. M. Ahsanullah, The generalized order statistics and a characteristic property of the exponential distribution. Pak. J. Statist., 11(3)(1995) 215-218.

21. M. Ahsanullah, Generalized order statistics from exponential distribution. Journal of Statistical Planning and Inference, 85(2000) 85-91.

22. M. Maswadah, Kernel Inference on the Inverse Weibull Distribution. The Korean Communications in Statistics, 13(3)(2006) 503- 512.

23. M. Maswadah, Kernel Inference on the Weibull Distribution. Proc. Third National Statistical Conference, (Lahore, Pakistan, 2007), 14, 77-86.

24. I. Abramson, On Bandwidth Variation in Kernel Estimates: A Square Root Law. Ann. Statist. 10(1982), 1217-1223.

25. A. Guillamon, J. Navarro and J. M. Ruiz, Kernel density estimation using weighted data. Commun. Statist.-Theory Meth., 27(9)(1998) 2123-2135.

26. P. Kulczycki, Parameter Identification Using Bayes and Kernel Approaches. Proceedings of the National Science Council ROC(A), 23(2)(1999), 205-213.

27. L. Bordes, Non-parametric estimation under progressive censoring. J. Statist. Plann. Inference, 119(2004), 171-189. 